\title{
Wave-Passage Effect of Earthquake Loadings on Long Structures
}

\author{
Ding Wang*,* Yueliang $\mathrm{Li}^{*, \S}$, Shengwang $\mathrm{Hao}^{*, \dagger,}$ and Dahai Zhao,\| \\ *School of Civil Engineering 8 Mechanics \\ Yanshan University, Qinhuangdao, P. R. China \\ ${ }^{\dagger}$ The State Key Laboratory of Nonlinear Mechanics \\ Institute of Mechanics, Chinese Academy of Science \\ Beijing, P. R. China \\ twangding@ysu.edu.cn \\ \$13933793164@163.com \\ "hsw@ysu.edu.cn \\ "zhaodh@ysu.edu.com
}

Received 9 November 2014

Accepted 15 June 2015

Published 23 July 2015

\begin{abstract}
The wave-passage effect of earthquake loadings on long-span structures is studied through use of a multiply-supported single-degree-of-freedom (SDOF) system excited by traveling seismic ground motions. The absolute acceleration response of the SDOF system is represented in the analytical form in the time domain. The frequency-domain analysis results indicate that the wave-passage effect may reduce the absolute acceleration response and the earthquake loading acting on the multiply-supported SDOF system. Further, for different velocities of wave-passage, the response spectra are calculated to represent the reduction of the maximum earthquake loading on the long-span system caused by the wave-passage effect. The computation results of the response spectra indicate that the reduction of the maximum earthquake loading is fluctuant, but has a general tendency to decrease with the increase in the apparent wave velocity and the structural natural period.
\end{abstract}

Keywords: Long structure; wave-passage effect; seismic ground motion; response spectrum; earthquake loading.

\section{Introduction}

For long structures, such as pipelines, suspension and cable-stayed bridges, dams, tunnels and long buildings, the effect of spatial variation of seismic ground motions is important and should be considered in the dynamic analysis and design of the structure. $^{1,2}$ Studies on the realistic ground motions recorded by the dense seismograph arrays show that three phenomena are responsible for the spatial variation of

\$Corresponding author. 
seismic ground motions, namely, the wave-passage effect, the incoherence effect and the local effect. ${ }^{3}$ The wave-passage effect, caused by wave propagation, causes differences in the arrival times of the earthquake waves at different supports of a long structure. The incoherence effect reflects the loss of coherency of the seismic ground motions. A number of parametric coherency models have been proposed to describe the incoherence effect. ${ }^{4-9}$ The local effect is caused by variations in the local soil condition, which influences the amplitude and frequency content of the bedrock ground motions. Physical causes underlying these variations can be summarized as the seismic wave traveling effect, extended source effect, scattering effect and attenuation effect. ${ }^{10}$

The first recognized cause for the spatial variations of earthquake ground motions was the apparent wave propagation on the ground. Up to the present, a variety of studies have been focused on the wave-passage effect on long structures, such as dams, ${ }^{11,12}$ long-span bridges, ${ }^{1}$ and highway bridges. ${ }^{13}$ The results of these studies show that the effect of seismic wave propagation on the response of long structures is significant and complex. For instance, the linear finite element analysis of the Sariyar concrete gravity dam, Turkey, subject to the wave-passage ground motions, presented by Bayraktar et al., ${ }^{11}$ shows that the vertical and shear stresses in the foundation increase with decreasing propagation velocity, but, at a cross section close to the base, the vertical and shear stresses do not exhibit a consistent pattern. At present, the general rules of the wave-passage effects on the structural responses are still poorly understood.

Commonly, the structural response excited by the spatially variable seismic ground motion can be separated into the pseudo-static component and the dynamic component. The pseudo-static response, which is caused by deformation of the ground, only exists when the excitations are nonuniform, and depends, to a great extent, on the displacement time histories of the seismic ground motion. Studies show that, in some case, the pseudo-static response may significantly increase the internal forces of the structural members. ${ }^{14}$ The dynamic response excited by the spatially variable seismic ground motion is caused by the inertia of the structure, which is similar to that in the uniform-excitation situation. From Newton's second law of motion, the action of the earthquake can be treated as the dynamic loading inducing the structural absolute acceleration response. At present, the acceleration response spectrum, defined by the maximum absolute acceleration response of the single-degree-of freedom (SDOF) systems with different natural periods, is widely used to determine the maximum dynamic earthquake loading of a specific ground motion for the design of structures and thus may be sensitive to the wave-passage effect. ${ }^{15,17}$

In this paper, we focus on the wave-passage effect of the dynamic earthquake loading imposed on long structures. The absolute acceleration response of a SDOFsystem on the multiple supports is derived in the time and frequency domains. Based on the results of the theoretical derivation of the structural response, the acceleration 
response spectrum of the propagating seismic ground motion is calculated to reflect the wave-passage effect on the maximum dynamic earthquake loading.

The purposes of this paper are: (1) To analyze the dynamic response of the SDOF, multiply-supported system subjected to the seismic wave-passage excitation and (2) to show how the wave-passage effect influences the dynamic earthquake loading on spatially extended structures.

\section{The Model}

As shown in Fig. 1, the model considered herein is a SDOF structure of rigid mass $m$, supported by $N$ same mass-less elastic columns. The columns are parallely connected to the ground and have the same properties, whose stiffness and viscous damping coefficient are $k_{i}$ and $c_{i}, i=1,2, \ldots, N$. The total stiffness, $k$, and viscous damping coefficient, $c$, satisfy

$$
k=\sum_{i=1}^{N} k_{i}, \quad c=\sum_{i=1}^{N} c_{i} .
$$

Let $x_{i}$ denote the distance from the left support to the $i$ th support. The distance from the left support to the geometric center of the supports can be calculated as:

$$
D=\frac{\sum_{i=1}^{N} x_{i}}{N} .
$$

The distance from the geometric center to the $i$ th support is:

$$
X_{i}=x_{i}-D \text {. }
$$

It is obvious that the distances satisfy $\Sigma X_{i}=0$. In this paper, we use a coordinate system with its origin located at the geometric center of the supports, to describe the positions of the supports and the distance $X_{i}$ is the coordinate of the $i$ th support.

Considering the spatial variation of the ground motion, the absolute displacements of the support bases are different, as a result of the wave-passage. In this

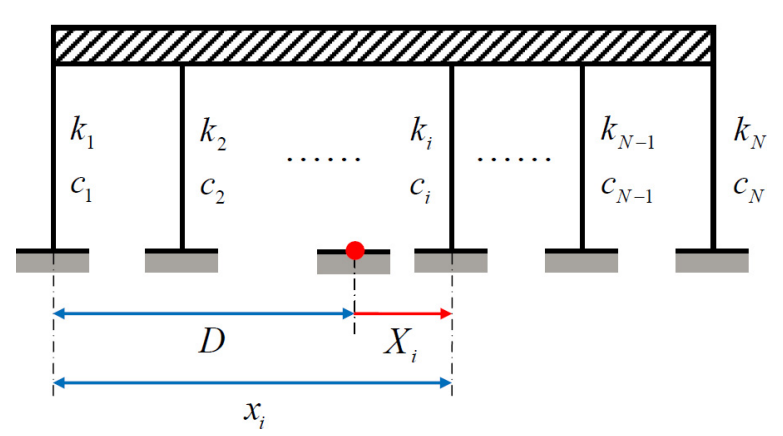

Fig. 1. Multiply-supported SDOF system. 


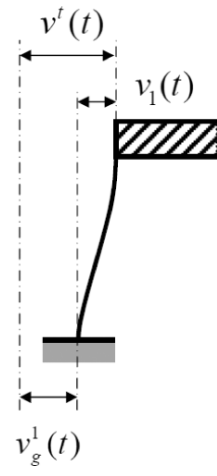

(a)

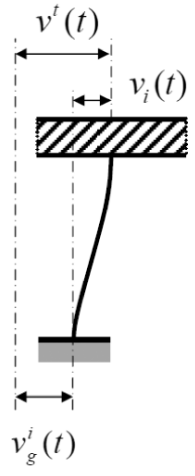

(b)

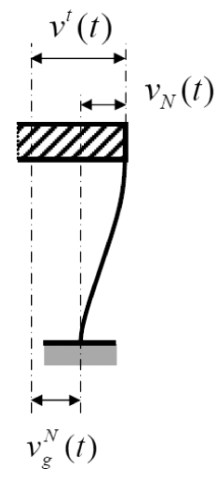

(c)

Fig. 2. Deformations of: (a) Left, (b) middle and (c) right, supports under the nonuniform seismic excitation.

paper, for simplicity, we just consider the horizontal component of the seismic wave and assume that the seismic wave propagates from left to right. Figure 2 shows the deformations of the supports, in which $v^{t}(t)$ is the absolute displacement response of the system, $v_{g}^{i}(t)$ is the spatial variable ground motion displacement at the $i$ th support base and $v_{i}(t)$ is the horizontal deformation of the $i$ th support. It is obvious that $v^{t}(t), v_{g}^{i}(t)$ and $v_{i}(t)$ satisfy

$$
v_{i}(t)=v^{t}(t)-v_{g}^{i}(t) \quad i=1,2, \ldots, N .
$$

The equation of motion of the SDOF system is

$$
m \cdot \ddot{v}^{t}(t)+\sum_{i=1}^{N} c_{i} \cdot \dot{v}_{i}(t)+\sum_{i=1}^{N} k_{i} \cdot v_{i}(t)=0
$$

Substituting Eq. (4) into Eq. (5), the motion equation can be represented by the absolute displacement of the system $v^{t}(t)$ as:

$$
m \cdot \ddot{v}^{t}(t)+\left(\sum_{i=1}^{N} c_{i}\right) \cdot \dot{v}^{t}(t)+\left(\sum_{i=1}^{N} k_{i}\right) \cdot v^{t}(t)=\sum_{i=1}^{N}\left[c_{i} \cdot \dot{v}_{g}^{i}(t)\right]+\sum_{i=1}^{N}\left[k_{i} \cdot v_{g}^{i}(t)\right]
$$

Considering the wave-passage effect, we assume that the site is uniform and the apparent wave velocity $c_{g}$ is invariable. As a result, the spatial variable ground motions at the support bases have the same shape function $v_{g}(t)$ but with different arriving delays $X_{i} / c_{g}$. Consequently, the spatial variable seismic ground motion $v_{g}^{i}(t)$ can be represented as:

$$
v_{g}^{i}(t)=v_{g}\left(t-\frac{X_{i}}{c_{g}}\right) .
$$




\section{Acceleration Response of the System in Time and Frequency Domains}

Equation (6) describes the motion of the multiply-supported SDOF system excited by the spatial variable seismic ground motion $v_{g}^{i}(t), i=1,2, \ldots, N$. In this section, a series solution of Eq. (6) will be presented, which indicates that the absolute displacement and acceleration responses excited by the traveling seismic wave can be expressed by the absolute displacement and acceleration responses excited by the corresponding uniform seismic wave. The frequency-domain analysis is also presented.

The apparent wave velocity is a key factor of the wave-passage effect. O'Rourke et al. estimated the apparent wave velocities of the 1971 San Fernando and the 1979 Imperial Valley earthquakes. ${ }^{18}$ For the San Fernando earthquake, the apparent wave velocity is in the range of $1.29-9.33 \mathrm{~km} / \mathrm{s}$. For the Imperial Valley earthquake, the apparent wave velocity is in the range of $1.34-20.9 \mathrm{~km} / \mathrm{s}$. Boissières and Vanmarcke estimated the lags of the spatial variable ground motions recorded by the SMART-1 array in Taiwan for 12 earthquake events, and indicated that the apparent passage velocity is in the range of $2.801-6.784 \mathrm{~km} / \mathrm{s} .{ }^{19}$ Hence, to most extended structures, the assumption is reasonable that $X_{i} / c_{g}$ is small enough in calculating the Taylor's expansion of Eq. (7). Mathematically, the Taylor's expansion for a general function $f(y)$ is

$$
f(y)=\sum_{l=0}^{+\infty} \frac{f^{(l)}\left(y_{0}\right)}{l !}\left(y-y_{0}\right)^{l}
$$

Taking $y=t-X_{i} / c_{g}$ and $y_{0}=t$ for Eq. (8), the Taylor's expansion of Eq. (7) can be expressed as:

$$
\begin{aligned}
v_{g}^{i}(t) & =v_{g}\left(t-\frac{X_{i}}{c_{g}}\right)=\sum_{l=0}^{+\infty} \frac{v_{g}^{(l)}(t)}{l !} \cdot\left(-\frac{X_{i}}{c_{g}}\right)^{l} \\
& =\sum_{l=0}^{+\infty}(-1)^{l} \cdot \frac{1}{l !} \cdot\left(\frac{X_{i}}{c_{g}}\right)^{l} \cdot v_{g}^{(l)}(t) .
\end{aligned}
$$

Substitute Eq. (9) into Eq. (6) yields

$$
\begin{aligned}
& m \cdot \ddot{v}^{t}(t)+\left(\sum_{i=1}^{N} c_{i}\right) \cdot \dot{v}^{t}(t)+\left(\sum_{i=1}^{N} k_{i}\right) \cdot v^{t}(t) \\
& =\sum_{l=0}^{+\infty}(-1)^{l} \cdot \frac{1}{l !}\left\{\sum_{i=1}^{N}\left[c_{i} \cdot\left(\frac{X_{i}}{c_{g}}\right)^{l}\right] \cdot v_{g}^{(l+1)}(t)+\sum_{i=1}^{N}\left[k_{i} \cdot\left(\frac{X_{i}}{c_{g}}\right)^{l}\right] \cdot v_{g}^{(l)}(t)\right\}
\end{aligned}
$$


As mentioned above, the column supports of the structural model have the same properties, namely,

$$
c_{i}=\frac{c}{N}, \quad k_{i}=\frac{k}{N} \quad i=1,2, \ldots, N
$$

Substituting Eq. (11) into Eq. (10), the equation of motion of the system can be simplified as:

$$
\begin{aligned}
& m \cdot \ddot{v}^{t}(t)+c \cdot \dot{v}^{t}(t)+k \cdot v^{t}(t) \\
& \quad=\sum_{l=0}^{+\infty}(-1)^{l} \cdot \frac{1}{l !} \cdot \frac{1}{N} \sum_{i=1}^{N}\left(\frac{X_{i}}{c_{g}}\right)^{l} \cdot\left\{c \cdot \dot{v}_{g}(t)+k \cdot v_{g}(t)\right\}^{(l)} .
\end{aligned}
$$

Equation (12) is a linear ordinary differential equation. Hence, the absolute displacement response $v^{t}(t)$ can be represented as:

$$
v^{t}(t)=\sum_{l=0}^{+\infty} v_{l}^{t}(t)
$$

in which $v_{l}^{t}(t)$ is the solution of the linear ordinary differential equation

$$
\begin{aligned}
& m \cdot \ddot{v}_{l}^{t}(t)+c \cdot \dot{v}_{l}^{t}(t)+k \cdot v_{l}^{t}(t) \\
& \quad=(-1)^{l} \cdot \frac{1}{l !} \cdot \frac{1}{N} \sum_{i=1}^{N}\left(\frac{X_{i}}{c_{g}}\right)^{l} \cdot\left\{c \cdot \dot{v}_{g}(t)+k \cdot v_{g}(t)\right\}^{(l)} \quad l=0,1,2, \ldots
\end{aligned}
$$

Particularly, for $l$ equal to 0, Eq. (14) becomes

$$
m \cdot \ddot{v}_{0}^{t}(t)+c \cdot \dot{v}_{0}^{t}(t)+k \cdot v_{0}^{t}(t)=c \cdot \dot{v}_{g}(t)+k \cdot v_{g}(t) .
$$

It should be noted that Eq. (15) has the same form as the equation of motion of the SDOF system excited by the uniform seismic ground motion $v_{g}(t)$. That means the solution of Eq. (15) is the absolute displacement response of the multiply-supported SDOF system under the uniform seismic excitation $v_{s}^{t}(t)$, i.e.

$$
v_{0}^{t}(t)=v_{s}^{t}(t)
$$

Equation (14) is a homogenous linear ordinary differential equation and the solution $v_{l}^{t}(t)$ can be represented by $v_{s}^{t}(t)$ as:

$$
v_{l}^{t}(t)=(-1)^{l} \cdot \frac{1}{l !} \cdot \sum_{i=1}^{N}\left(\frac{X_{i}}{c_{g}}\right)^{l} \cdot \frac{d^{l}}{d t^{l}} \cdot v_{s}^{t}(t)
$$

From Eqs. (13) and (17), the absolute displacement response of the multiplysupported SDOF system excited by the traveling seismic wave is

$$
v^{t}(t)=\sum_{l=0}^{+\infty} v_{l}^{t}(t)=\left\{1+\frac{1}{N} \cdot \sum_{l=1}^{+\infty}\left[(-1)^{l} \cdot \frac{1}{l !} \cdot \sum_{i=1}^{N}\left(\frac{X_{i}}{c_{g}}\right)^{l} \cdot \frac{d^{l}}{d t^{l}}\right]\right\} \cdot v_{s}^{t}(t)
$$


and the absolute acceleration response is

$$
a^{t}(t)=\ddot{v}^{t}(t)=\left\{1+\frac{1}{N} \cdot \sum_{l=1}^{+\infty}\left[(-1)^{l} \cdot \frac{1}{l !} \cdot \sum_{i=1}^{N}\left(\frac{X_{i}}{c_{g}}\right)^{l} \cdot \frac{d^{l}}{d t^{l}}\right]\right\} \cdot a_{s}^{t}(t),
$$

in which $a_{s}^{t}(t)$ is the absolute acceleration response of the system under the uniform ground motion excitation $v_{g}(t)$. Equations (18) and (19) show the connection between the system responses excited by the uniform seismic wave and by the traveling seismic wave. As it is well known, the absolute acceleration response reflects the dynamic earthquake loading on the structure. Hence, Eq. (19) indicates the connection between the dynamic earthquake loadings produced by the uniform seismic wave and by the traveling seismic wave.

In some cases, it is convenient to represent formula (19) as:

$$
a^{t}(t)=\sum_{l=0}^{+\infty} G_{l}^{t}(t)-\sum_{l=0}^{+\infty} H_{l}^{t}(t)
$$

in which

$$
\begin{aligned}
& G_{l}^{t}(t)=\frac{1}{N} \cdot \frac{1}{(2 l) !} \cdot \sum_{i=1}^{N}\left(\frac{X_{i}}{c_{g}}\right)^{2 l} \cdot \frac{d^{2 l}}{d t^{2 l}} a_{s}^{t}(t), \\
& H_{l}^{t}(t)=\frac{1}{N} \cdot \frac{1}{(2 l+1) !} \cdot \sum_{i=1}^{N}\left(\frac{X_{i}}{c_{g}}\right)^{2 l+1} \cdot \frac{d^{2 l+1}}{d t^{2 l+1}} a_{s}^{t}(t) .
\end{aligned}
$$

Particularly, when the distribution of the supports is symmetrical, the coordinates $X_{i}, i=1,2, \ldots, N$, satisfy

$$
\sum_{i=0}^{N} X_{i}^{2 l+1}=0 \quad l=0,1,2, \ldots
$$

Substituting Eq. (23) into Eq. (22), we get

$$
H_{l}^{t}(t)=0 \quad l=0,1,2, \ldots
$$

Hence, the acceleration response (20) can be simplified as:

$$
a^{t}(t)=\sum_{l=0}^{+\infty} G_{l}^{t}(t)=\sum_{l=0}^{+\infty}\left[\frac{1}{N} \cdot \frac{1}{(2 l) !} \cdot \sum_{i=1}^{N}\left(\frac{X_{i}}{c_{g}}\right)^{2 l} \cdot \frac{d^{2 l}}{d t^{2 l}} a_{s}^{t}(t)\right] .
$$

Equation (20) gives the acceleration response of the system in the time domain. The frequency-domain characteristics of the system response can be represented by the Fourier spectrum of Eq. (20). The Fourier transforms of Eqs. (21) and (22) are

$$
\mathscr{F}\left[G_{l}^{t}(t)\right]=\int_{-\infty}^{+\infty}\left[\frac{1}{N} \cdot \frac{1}{(2 l) !} \cdot \sum_{i=1}^{N}\left(\frac{X_{i}}{c_{g}}\right)^{2 l} \cdot \frac{d^{2 l}}{d t^{2 l}} a_{s}^{t}(t)\right] \cdot e^{-j \omega \pi} d x
$$


D. Wang et al.

$$
\begin{aligned}
& =\frac{1}{N} \cdot \frac{1}{(2 l) !} \cdot \sum_{i=1}^{N}\left(\frac{X_{i}}{c_{g}}\right)^{2 l} \cdot(-j \omega)^{2 l} \int_{-\infty}^{+\infty} a_{s}^{t}(t) \cdot e^{-j \omega \pi} d x \\
& =\frac{1}{N} \cdot \sum_{i=1}^{N}\left[\frac{(-1)^{l}}{(2 l) !} \cdot\left(\frac{\omega X_{i}}{c_{g}}\right)^{2 l}\right] \cdot \mathscr{F}\left[a_{s}^{t}(t)\right]
\end{aligned}
$$

and

$$
\begin{aligned}
\mathscr{F}\left[H_{l}^{t}(t)\right] & =\int_{-\infty}^{+\infty}\left[\frac{1}{N} \cdot \frac{1}{(2 l+1) !} \cdot \sum_{i=1}^{N}\left(\frac{X_{i}}{c_{g}}\right)^{2 l+1} \cdot \frac{d^{2 l+1}}{d t^{2 l+1}} a_{s}^{t}(t)\right] \cdot e^{-j \omega \pi} d x \\
& =\frac{1}{N} \cdot \frac{1}{(2 l+1) !} \cdot \sum_{i=1}^{N}\left(\frac{X_{i}}{c_{g}}\right)^{2 l+1} \cdot(-j \omega)^{2 l+1} \int_{-\infty}^{+\infty} a_{s}^{t}(t) \cdot e^{-j \omega \pi} d x \\
& =-\frac{j}{N} \cdot \sum_{i=1}^{N}\left[\frac{(-1)^{l}}{(2 l+1) !} \cdot\left(\frac{\omega X_{i}}{c_{g}}\right)^{2 l+1}\right] \cdot \mathscr{F}\left[a_{s}^{t}(t)\right]
\end{aligned}
$$

where $j=\sqrt{-1}$. By the power series expansions of the trigonometric functions

$$
\cos x=\sum_{l=0}^{+\infty}(-1)^{n} \cdot \frac{x^{2 n}}{(2 n) !}, \quad \sin x=\sum_{l=0}^{+\infty}(-1)^{n} \cdot \frac{x^{2 n+1}}{(2 n+1) !},
$$

the Fourier transform of Eq. (20) can be calculated as:

$$
\begin{aligned}
\mathscr{F}\left[a^{t}(t)\right]= & \sum_{l=0}^{+\infty} \mathscr{F}\left[G_{l}^{t}(t)\right]-\sum_{l=0}^{+\infty} \mathscr{F}\left[H_{l}^{t}(t)\right] \\
= & \frac{1}{N} \cdot \sum_{i=1}^{N}\left[\sum_{l=0}^{+\infty} \frac{(-1)^{l}}{(2 l) !}\left(\frac{\omega X_{i}}{c_{g}}\right)^{2 l}\right] \cdot \mathscr{F}\left[a_{s}^{t}(t)\right] \\
& +\frac{j}{N} \sum_{i=1}^{N}\left[\sum_{l=0}^{+\infty} \frac{(-1)^{l}}{(2 l+1) !}\left(\frac{\omega X_{i}}{c_{g}}\right)^{2 l+1}\right] \cdot \mathscr{F}\left[a_{s}^{t}(t)\right] \\
= & \frac{1}{N} \sum_{i=1}^{N}\left[\cos \left(\frac{\omega X_{i}}{c_{g}}\right)+j \sin \left(\frac{\omega X_{i}}{c_{g}}\right)\right] \cdot \mathscr{F}\left[a_{s}^{t}(t)\right] .
\end{aligned}
$$

From Eq. (29), the Fourier amplitude spectrum of $a^{t}(t)$ is

$$
A^{t}(\omega)=\left|\mathscr{F}\left[a^{t}(t)\right]\right|=\frac{1}{N} \sqrt{\left[\sum_{i=1}^{N} \cos \left(\frac{\omega X_{i}}{c_{g}}\right)\right]^{2}+\left[\sum_{i=1}^{N} \sin \left(\frac{\omega X_{i}}{c_{g}}\right)\right]^{2}} \cdot A_{s}^{t}(\omega),
$$

in which $A_{s}^{t}(\omega)=\left|\mathscr{F}\left[a_{s}^{t}(t)\right]\right|$ is the Fourier amplitude spectrum of $a_{s}^{t}(t)$. Equation (30) shows the frequency-domain connection of the absolute acceleration responses excited by the uniform seismic wave and by the traveling seismic wave. Further, the 
ratio of $A^{t}(\omega)$ to $A_{s}^{t}(\omega)$ can be calculated as:

$$
\frac{A^{t}(\omega)}{A_{s}^{t}(\omega)}=\frac{1}{N} \sqrt{\left[\sum_{i=1}^{N} \cos \left(\frac{\omega X_{i}}{c_{g}}\right)\right]^{2}+\left[\sum_{i=1}^{N} \sin \left(\frac{\omega X_{i}}{c_{g}}\right)\right]^{2}},
$$

where

$$
\begin{aligned}
{\left[\sum_{i=1}^{N} \cos \left(\frac{\omega X_{i}}{c_{g}}\right)\right]^{2} } & =\left[\sum_{i=1}^{N} \cos \left(\frac{\omega X_{i}}{c_{g}}\right)\right] \cdot\left[\sum_{k=1}^{N} \cos \left(\frac{\omega X_{k}}{c_{g}}\right)\right] \\
& =\sum_{i=1}^{N}\left[\cos \left(\frac{\omega X_{i}}{c_{g}}\right) \cdot\left\{\sum_{k=1}^{N} \cos \left(\frac{\omega X_{k}}{c_{g}}\right)\right\}\right] \\
& =\sum_{i=1}^{N} \sum_{k=1}^{N}\left[\cos \left(\frac{\omega X_{i}}{c_{g}}\right) \cdot \cos \left(\frac{\omega X_{k}}{c_{g}}\right)\right]
\end{aligned}
$$

and, in the same way,

$$
\left[\sum_{i=1}^{N} \sin \left(\frac{\omega X_{i}}{c_{g}}\right)\right]^{2}=\sum_{i=1}^{N} \sum_{k=1}^{N}\left[\sin \left(\frac{\omega X_{i}}{c_{g}}\right) \cdot \sin \left(\frac{\omega X_{k}}{c_{g}}\right)\right] .
$$

Substituting Eqs. (32) and (33) into Eq. (31), we get

$$
\frac{A^{t}(\omega)}{A_{s}^{t}(\omega)}=\frac{1}{N} \sqrt{\sum_{i=1}^{N} \sum_{k=1}^{N}\left[\cos \left(\frac{\omega X_{i}}{c_{g}}\right) \cos \left(\frac{\omega X_{k}}{c_{g}}\right)+\sin \left(\frac{\omega X_{i}}{c_{g}}\right) \sin \left(\frac{\omega X_{k}}{c_{g}}\right)\right]} .
$$

According to the angle difference identity

$$
\cos (\alpha-\beta)=\cos \alpha \cos \beta+\sin \alpha \sin \beta \quad \text { for } \forall \alpha, \beta .
$$

Equation (34) can be written as:

$$
\frac{A^{t}(\omega)}{A_{s}^{t}(\omega)}=\frac{1}{N} \sqrt{\sum_{i=1}^{N} \sum_{k=1}^{N} \cos \left(\frac{\omega X_{i}}{c_{g}}-\frac{\omega X_{k}}{c_{g}}\right)}
$$

In Eq. (36), the number of the cosine terms of

$$
\sum_{i=1}^{N} \sum_{k=1}^{N} \cos \left(\frac{\omega X_{i}}{c_{g}}-\frac{\omega X_{k}}{c_{g}}\right)
$$

is $N^{2}$. Hence,

$$
\frac{A^{t}(\omega)}{A_{s}^{t}(\omega)}=\frac{1}{N} \sqrt{\sum_{i=1}^{N} \sum_{k=1}^{N} \cos \left(\frac{\omega X_{i}}{c_{g}}-\frac{\omega X_{k}}{c_{g}}\right)} \leq \frac{1}{N} \sqrt{N^{2}}=1 .
$$


Equation (37) shows that the Fourier amplitude spectrum of the wave-passage excited acceleration response $a^{t}(t)$ is not larger than the Fourier amplitude spectrum of the uniform excited acceleration response $a_{s}^{t}(t)$, namely,

$$
A^{t}(\omega) \leq A_{s}^{t}(\omega)
$$

The preceding equation indicates that, in the frequency domain, the wave-passage effect reduces the absolute acceleration response and, as a result, reduces the dynamic earthquake loading on the multiply-supported SDOF system.

\section{Response Spectrum Considering Seismic Wave-Passage Effect}

Perhaps, the response spectrum is the most basic tool used in earthquake engineering and structural seismic design. ${ }^{20,21}$ For a particular seismic ground motion, the response spectrum is given by the maximum absolute value of the displacement, velocity or acceleration of a SDOF oscillator with specified damping and natural period. The absolute acceleration response spectrum represents the maximum dynamic earthquake loading on the structure. However, the SDOF oscillator used in the computation of the response spectrum is singly supported, which means it is inapplicable for the studies on the spatial variation of seismic ground motions. In this section, for the multiply-supported SDOF system, the absolute acceleration response spectrum considering the wave-passage effect is proposed to reflect the maximum dynamic earthquake loading caused by the traveling seismic wave.

The absolute acceleration response spectrum considering the wave-passage effect can be defined as:

$$
S_{a, c_{g}}(T, \xi, \mathbf{X})=\max \left|a^{t}(t)\right|,
$$

where $T$ is the period, $\xi$ is the damping ratio and $\mathbf{X}$ is the support-location vector, defined as:

$$
\mathbf{X}=\left[X_{1}, X_{2}, \ldots, X_{N}\right]
$$

The key step to calculating $S_{a, c_{g}}(T, \xi, \mathbf{X})$ is the computation of $a^{t}(t)$. In this paper, from Eq. (20), $a^{t}(t)$ is calculated by the first $M+1$ terms of $\left\{G_{l}^{t}(t)\right\}$ and the first $M+1$ terms of $\left\{H_{l}^{t}(t)\right\}$ as:

$$
a^{t}(t) \approx \sum_{l=0}^{M} G_{l}^{t}(t)-\sum_{l=0}^{M} H_{l}^{t}(t)
$$

where the number of terms is determined by the following conditions:

$$
\max \left(\frac{\left|G_{M-1}^{t}(t)\right|}{\left|G_{0}^{t}(t)\right|}, \frac{\left|H_{M-1}^{t}(t)\right|}{\left|H_{0}^{t}(t)\right|}\right) \geq \varepsilon, \quad \max \left(\frac{\left|G_{M}^{t}(t)\right|}{\left|G_{0}^{t}(t)\right|}, \frac{\left|H_{M}^{t}(t)\right|}{\left|H_{0}^{t}(t)\right|}\right)<\varepsilon,
$$

where $\varepsilon$ is a chosen threshold. The convergence of Eq. (41) is controlled by the value of $\varepsilon$. In fact, Eq. (42) requires that, for the $M$ th term, the ratios of $\left|G_{M}^{t}(t)\right|$ to $\left|G_{0}^{t}(t)\right|$ and of $\left|H_{M}^{t}(t)\right|$ to $\left|H_{0}^{t}(t)\right|$ should both be less than $\varepsilon$ throughout the response process. 


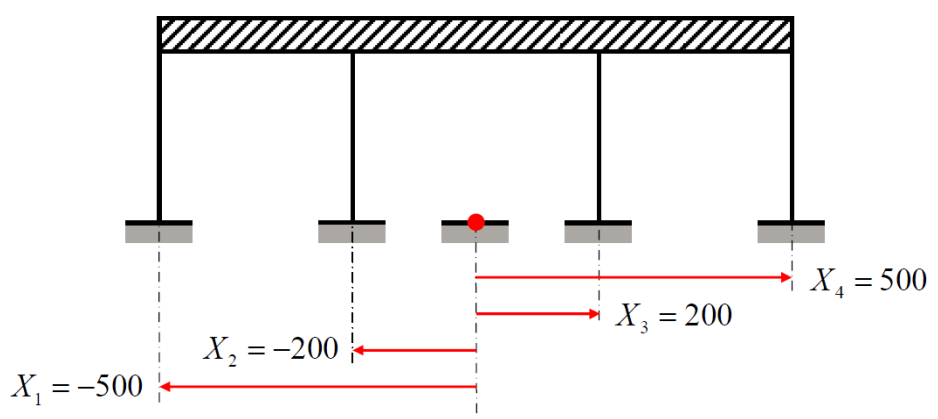

Fig. 3. Four-supported SDOF system which support-location vector is $X=[-500,-200,200,500]$.

Hence, for a small value of $\varepsilon$, it is reasonable that the terms with $l>M$ are small enough to be neglected and Eq. (41) is suitable for calculating the acceleration response $a^{t}(t)$.

A numerical example is presented to show the process of computation of the response spectrum. Figure 3 shows a SDOF system with four supports exposed to a horizontal wave-passage earthquake excitation. The damping ratio of the system is 0.02 and the support-location vector is

$$
\mathbf{X}=[-500,-200,200,500] .
$$

In this example, the $\mathrm{N}-\mathrm{S}$ component of the ground motion recorded at the El Centro station in the 1940 Imperial Valley earthquake, as shown in Fig. 4, is used as the base excitation, for which the apparent wave velocity is $2000 \mathrm{~m} / \mathrm{s}$. The absolute acceleration response excited by the uniform earthquake wave is calculated by the Newmark- $\beta$ method. Due to the symmetry of the support distribution, $H_{l}^{t}(t)$ equals 0 and the absolute acceleration response excited by the traveling earthquake wave can be calculated from Eq. (25).

The acceleration responses of the system with natural periods $T=0.2,0.4,0.6$, $0.8,1.0,1.2,1.5$ and $2.0 \mathrm{~s}$ are computed. The threshold in Eq. (42) is chosen as 0.001 . The details of the response computation are represented in Table 1. Figure 5 shows

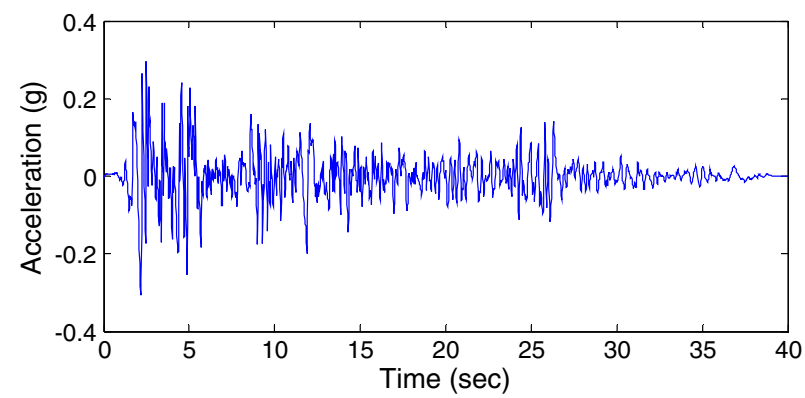

Fig. 4. Time history recorded at the El Centro station during the 1940 Imperial Valley earthquake in the N-S direction. 
D. Wang et al.

Table 1. Acceleration response computation of the multiply-supported, SDOF systems with different natural periods.

\begin{tabular}{lccc}
\hline No. & $T / s^{-1}$ & $2 M+2$ & $\max \left[\left|G_{M-1}^{t}(t) / G_{0}^{t}(t)\right|,\left|H_{M-1}^{t}(t) / H_{0}^{t}(t)\right|\right]\left(\times 10^{-4}\right)$ \\
\hline 1 & 0.2 & 36 & 2.4902 \\
2 & 0.4 & 34 & 5.1163 \\
3 & 0.6 & 34 & 2.2690 \\
4 & 0.8 & 32 & 9.3048 \\
5 & 1.0 & 32 & 6.3295 \\
6 & 1.2 & 32 & 6.5642 \\
7 & 1.5 & 34 & 1.6124 \\
8 & 2.0 & 34 & 5.8142 \\
\hline
\end{tabular}

the comparison between the acceleration responses caused by the uniform excitation and by the traveling excitation. The results indicate that the wave-passage effect significantly decreases the absolute acceleration responses of the system with natural periods less than $1 \mathrm{~s}$. For the system with natural periods larger than $1 \mathrm{~s}$, the effect of the wave propagation on the responses gradually decreases with the increase of the natural period.

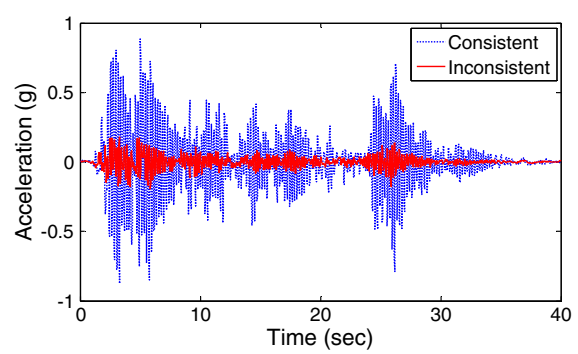

(a)

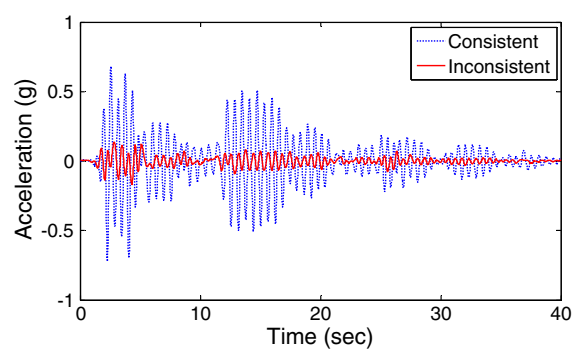

(c)

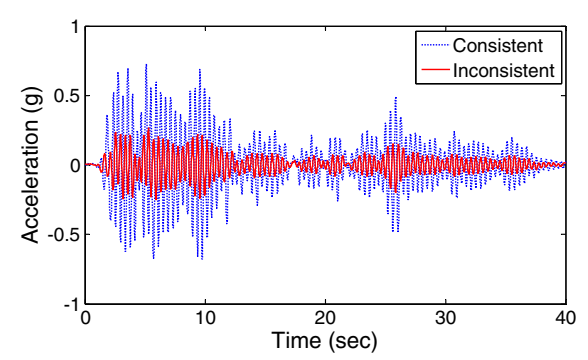

(b)

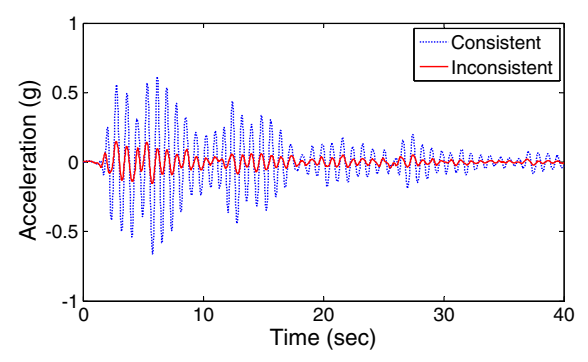

(d)

Fig. 5. Acceleration responses excited by the traveling seismic wave and by the uniform seismic wave for different structural natural periods. (a) $T=0.2 \mathrm{~s}$, (b) $T=0.4 \mathrm{~s}$, (c) $T=0.6 \mathrm{~s}$, (d) $T=0.8 \mathrm{~s}$, (e) $T=1.0 \mathrm{~s}$, (f) $T=1.2 \mathrm{~s},(\mathrm{~g}) T=1.5 \mathrm{~s}$ and (h) $T=2.0 \mathrm{~s}$. 


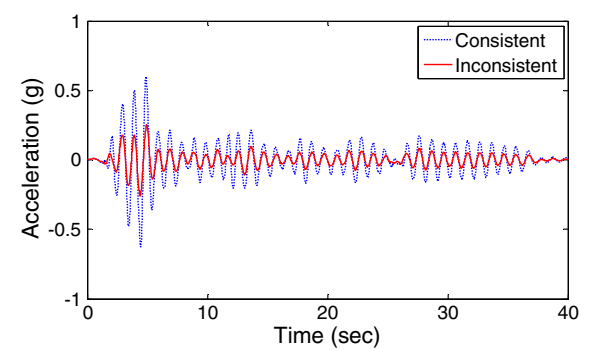

(e)

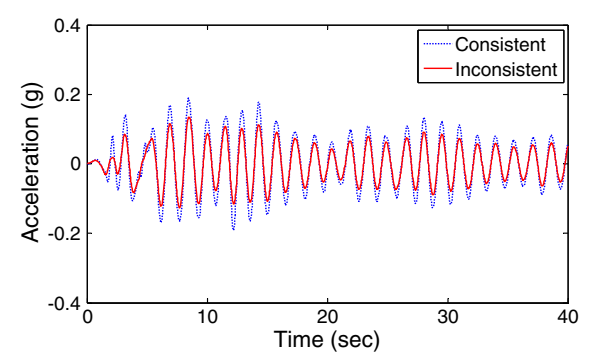

$(\mathrm{g})$

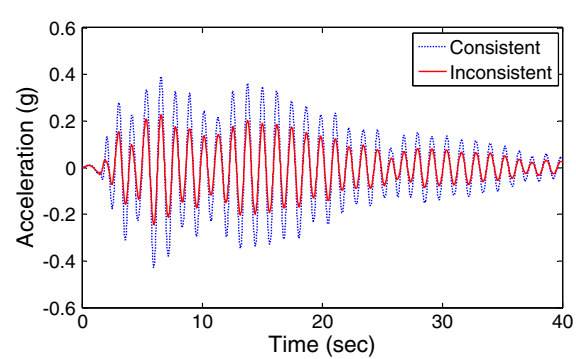

(f)

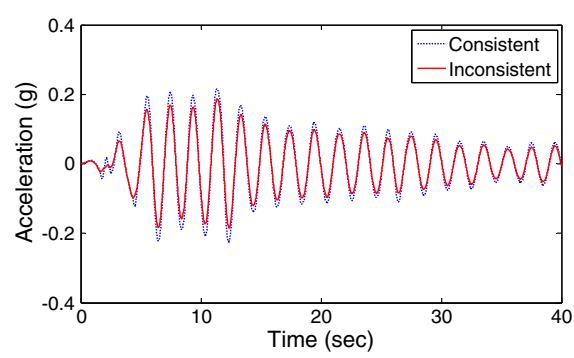

(h)

Fig. 5. (Continued)

The value of the threshold $\varepsilon$ controls the convergence of Eq. (41). In this numerical example, the value of $\varepsilon$ is 0.001 . Tables 2 and 3 show the effects of the different values of $\varepsilon$ on the computation of the acceleration response for the multiplysupported systems with natural periods of 0.4 and $1.2 \mathrm{~s}$, respectively. The peak value and the total energy, which is defined as

$$
\text { Total energy }=\int_{0}^{D}\left[a^{t}(t)\right]^{2} \cdot \mathrm{d} t,
$$

of the absolute acceleration response are used to measure the convergence of the computation result. In Eq. (44), $D$ is the duration of the response. Tables 2 and 3 both show that, for $\varepsilon<0.01$, the peak values and the total energies of the calculated acceleration responses are identical. Hence, for $\varepsilon=0.001$, Equation (41) is convergent and available to calculate the response in this numeral example. For general applications, we suggest that the value of $\varepsilon$ should be chosen small enough to make the peak value and the total energy of the response stable.

When the natural period of the system changes continuously, we get the wave-passage response spectrum with the support-location vector $\mathbf{X}=[-500$, $-200,200,500]$, which is defined by Eq. (39), as shown in Fig. 6(a). A more intuitive tool for reflecting the wave-passage effect is the response spectral ratio, 
Table 2. Effect of $\varepsilon$ on the PGA and the total energy of the acceleration response of the multiply-supported SDOF system $(T=0.4 \mathrm{~s})$.

\begin{tabular}{lccc}
\hline$\varepsilon$ & $2 M+2$ & PGA $(\mathrm{g})$ & Total energy $\left(\mathrm{g}^{2} \cdot \mathrm{s}\right)$ \\
\hline 0.1 & 28 & 0.2600 & 0.2264 \\
0.05 & 30 & 0.2634 & 0.2263 \\
0.01 & 32 & 0.2629 & 0.2263 \\
0.005 & 32 & 0.2629 & 0.2263 \\
0.004 & 32 & 0.2629 & 0.2263 \\
0.003 & 34 & 0.2629 & 0.2263 \\
0.002 & 34 & 0.2629 & 0.2263 \\
0.001 & 34 & 0.2629 & 0.2263 \\
0.0001 & 36 & 0.2629 & 0.2263 \\
\hline
\end{tabular}

defined as:

$$
C_{a, c_{g}}(T, \xi, \mathbf{X})=\frac{S_{a, c_{g}}(T, \xi, \mathbf{X})}{S_{a,+\infty}(T, \xi, \mathbf{X})}=\frac{S_{a, c_{g}}(T, \xi, \mathbf{X})}{S_{a}(T, \xi)}
$$

where $S_{a}(T, \xi)$ is the corresponding acceleration response spectrum of the uniform earthquake ground motion. Figure $6(\mathrm{~b})$ shows the response spectral ratio $C_{a, 2000}(T)$ of this numerical example.

In fact, the wave-passage acceleration response spectrum and the response spectral ratio, as shown in Fig. 6, indicate the wave-passage effect on the maximum dynamic earthquake loading imposed on the structure. The reduction of the maximum dynamic earthquake loading is remarkable in the range of $0-1 \mathrm{~s}$, and decreases in the range of $1-2 \mathrm{~s}$. For the range large than $2 \mathrm{~s}$, the wave-passage effect on the maximum dynamic earthquake loading is weak and can be ignored.

Generally, as indicated by the frequency-domain analysis of the acceleration response of the multiply-supported SDOF system, the wave-passage effect reduces the maximum dynamic earthquake loading on the structure. The wave-passage response spectrum and the response spectral ratio are the intuitive and convenient tools for evaluating the reduction of the maximum dynamic earthquake loading.

Table 3. Effect of $\varepsilon$ on the PGA and the total energy of the acceleration response of the multiply-supported SDOF system $(T=1.2 \mathrm{~s})$.

\begin{tabular}{lccc}
\hline$\varepsilon$ & $2 M+2$ & Peak acceleration $(\mathrm{g})$ & Total energy $\left(\mathrm{g}^{2} \cdot \mathrm{s}\right)$ \\
\hline 0.1 & 26 & 0.2439 & 0.3176 \\
0.05 & 28 & 0.2437 & 0.3176 \\
0.01 & 30 & 0.2437 & 0.3176 \\
0.005 & 30 & 0.2437 & 0.3176 \\
0.004 & 30 & 0.2437 & 0.3176 \\
0.003 & 32 & 0.2437 & 0.3176 \\
0.002 & 32 & 0.2437 & 0.3176 \\
0.001 & 32 & 0.2437 & 0.3176 \\
0.0001 & 36 & 0.2437 & 0.3176 \\
\hline
\end{tabular}




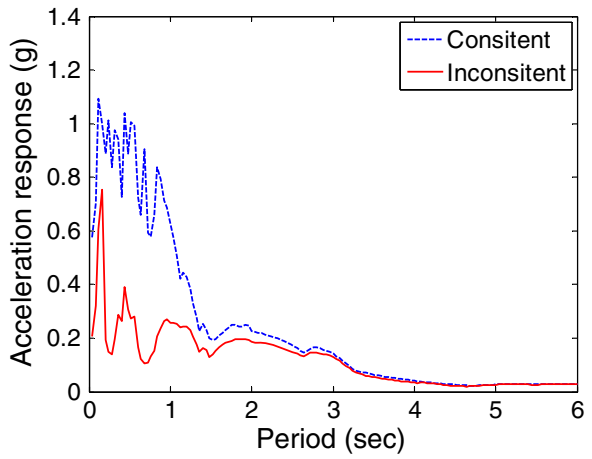

(a)

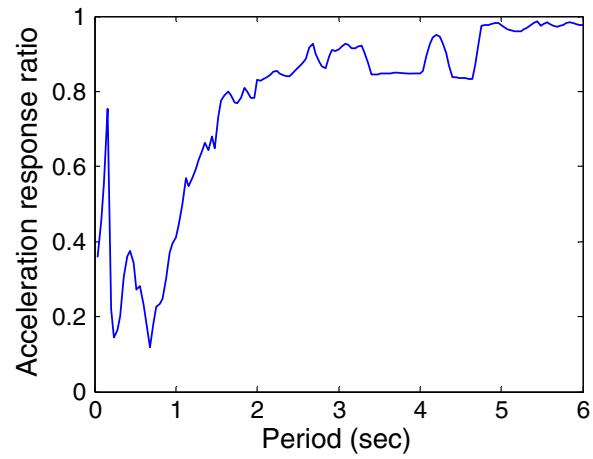

(b)

Fig. 6. (a) Response spectra and (b) response spectral ratios for $c g=2000 \mathrm{~m} / \mathrm{s}$.

\section{Effects of Apparent Wave Velocity}

Equation (30) indicates that the structural support locations and the apparent wave velocity are the key factors controlling the structural response reduction caused by the wave-passage effect. In most cases, the support locations are determined by the engineering geological conditions or the structural design requirements. Hence, in this section, we focus on the effects of the apparent wave velocity on the structural absolute acceleration response and the dynamic earthquake loading incurred.

The four-supported SDOF system, shown in Fig. 3, is again adopted in this example. The natural period of the system is $1 \mathrm{~s}$ and the damping ratio is 0.02 . Figure 7 shows the absolute acceleration responses of the system excited by the El Centro seismic ground motion given in Fig. 4 for different apparent wave velocities. The apparent wave velocities considered herein are in the range of $600-5000 \mathrm{~m} / \mathrm{s}$. The computation details are shown in Table 4. The calculation results indicate that

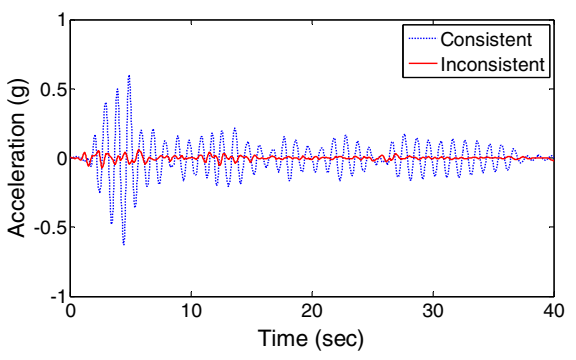

(a)

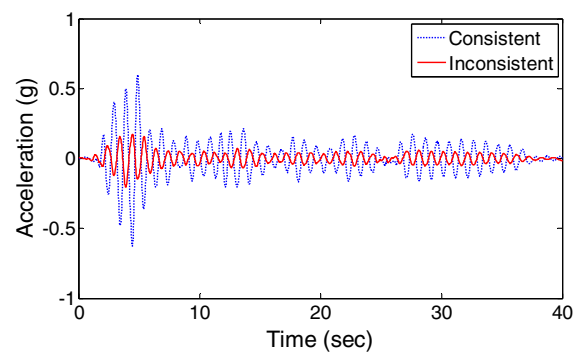

(b)

Fig. 7. Acceleration responses excited by the traveling seismic wave and by the uniform seismic wave for different apparent velocities. (a) $c_{g}=600 \mathrm{~m} / \mathrm{s}$, (b) $c_{g}=800 \mathrm{~m} / \mathrm{s}$, (c) $c_{g}=1000 \mathrm{~m} / \mathrm{s}$, (d) $c_{g}=1200 \mathrm{~m} / \mathrm{s}$, (e) $c_{g}=1400 \mathrm{~m} / \mathrm{s}$, (f) $c_{g}=1600 \mathrm{~m} / \mathrm{s}$, (g) $c_{g}=1800 \mathrm{~m} / \mathrm{s}, \quad(\mathrm{h}) c_{g}=2000 \mathrm{~m} / \mathrm{s}, \quad$ (i) $c_{g}=3000 \mathrm{~m} / \mathrm{s}$ and (j) $c_{g}=5000 \mathrm{~m} / \mathrm{s}$. 
D. Wang et al.

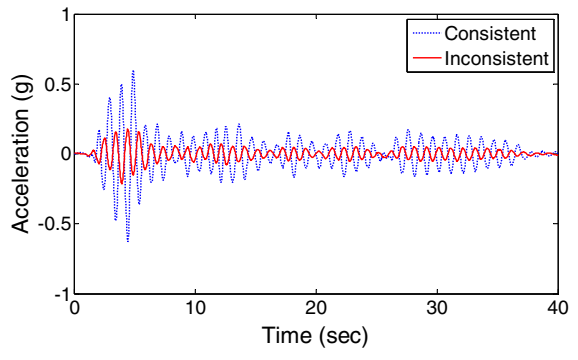

(c)

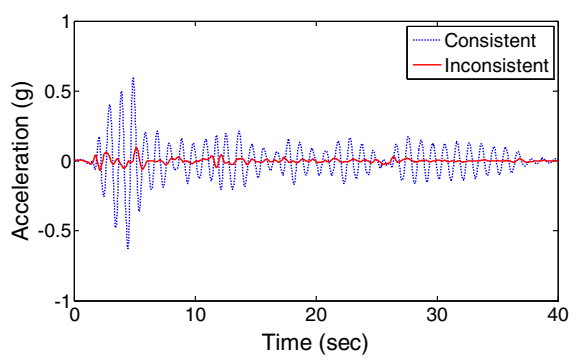

(e)

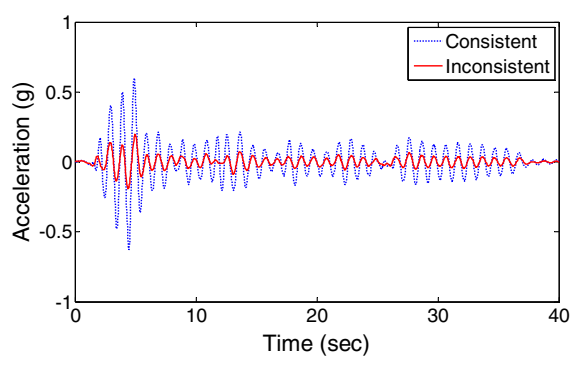

(g)

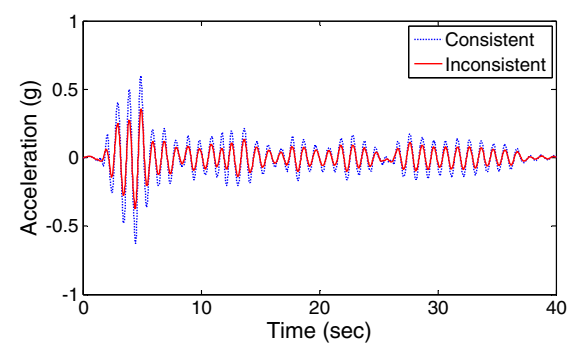

(i)

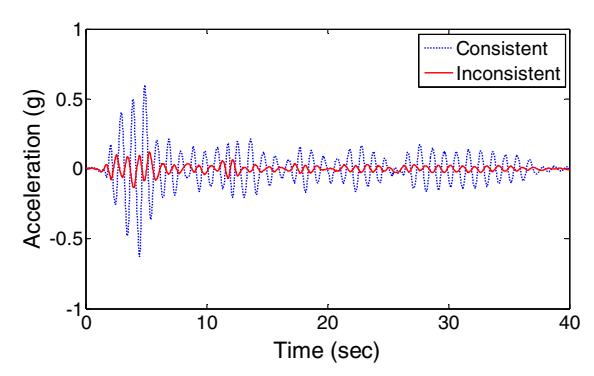

(d)

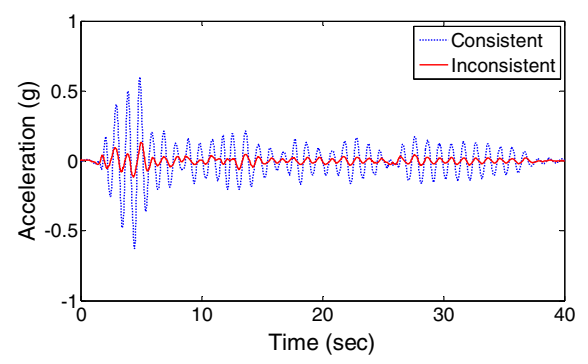

(f)

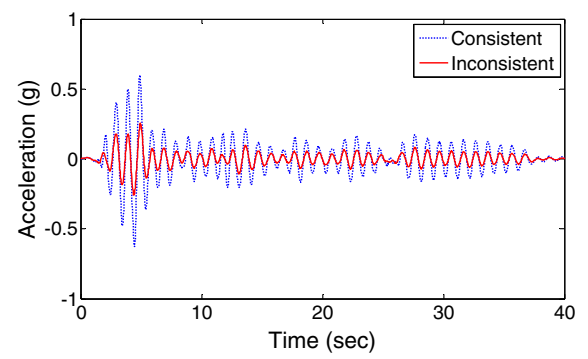

(h)

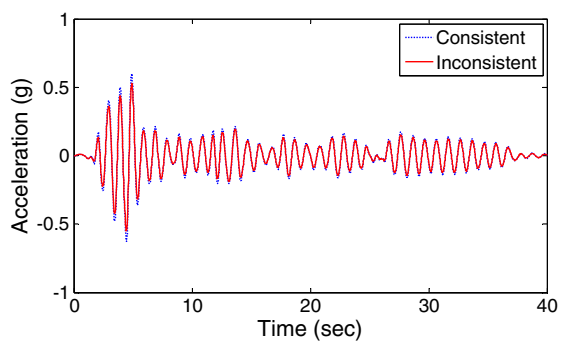

(j)

Fig. 7. (Continued) 
Table 4. Acceleration response computation of the multiply-supported, SDOF system for different apparent wave velocities.

\begin{tabular}{rccc}
\hline No. & $c_{g} / m \cdot s^{-1}$ & $2 M+2$ & $\max \left[\left|G_{M-1}^{t}(t) / G_{0}^{t}(t)\right|,\left|H_{M-1}^{t}(t) / H_{0}^{t}(t)\right|\right]\left(\times 10^{-4}\right)$ \\
\hline 1 & 600 & 110 & 9.5063 \\
2 & 800 & 82 & 8.5151 \\
3 & 1000 & 66 & 3.8594 \\
4 & 1200 & 54 & 8.1794 \\
5 & 1400 & 46 & 8.2160 \\
6 & 1600 & 40 & 8.3137 \\
7 & 1800 & 36 & 4.6248 \\
8 & 2000 & 32 & 6.3295 \\
9 & 3000 & 22 & 2.8157 \\
10 & 5000 & 14 & 1.8796 \\
\hline
\end{tabular}

the response reduction caused by the wave-passage effect exhibits a remarkable fluctuation. In the range of $c_{g}=600-1400 \mathrm{~m} / \mathrm{s}$, the response increases at first then decreases. For $c_{g}>1400 \mathrm{~m} / \mathrm{s}$, the response continuously increases and gradually reaches the uniform-excited level.

It is intuitive to define a function as:

$$
R\left(c_{g}\right)=\frac{\max \left[\left|a^{t}(t)\right|\right]}{\max \left[\left|a_{s}^{t}(t)\right|\right]},
$$

to represent the effect of the apparent wave velocity on the maximum dynamic earthquake loading on the structure. Figure 8 shows the computation results of $R\left(c_{g}\right)$ for the SDOF systems with different natural periods. The natural periods considered herein are $0.1,0.2,0.4,0.7,1.0$ and $3.0 \mathrm{~s}$. For the system with low natural periods, as shown in Figs. 8(a) and 8(b), the value $R$ fluctuates significantly in the low apparent

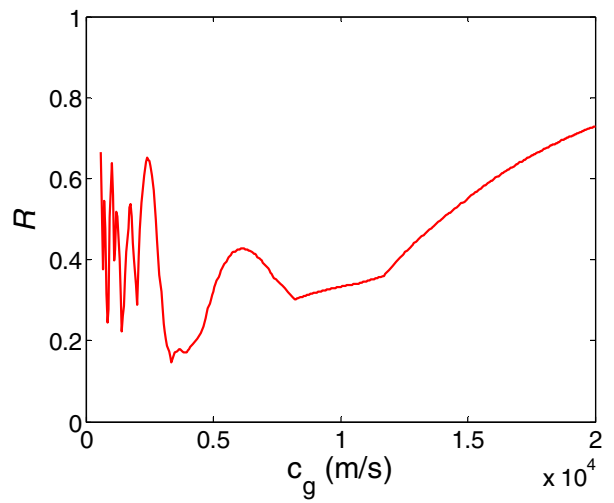

(a)

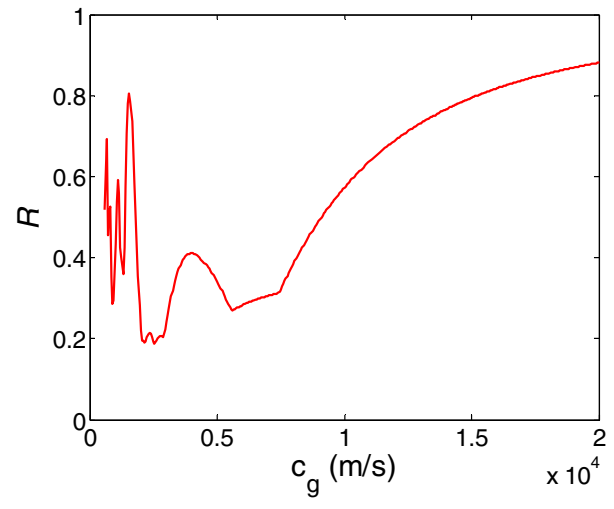

(b)

Fig. 8. Effect of the apparent wave velocity on the maximum dynamic earthquake loading on the SDOF system. (a) $T=0.1 \mathrm{~s}$, (b) $T=0.2 \mathrm{~s}$, (c) $T=0.4 \mathrm{~s}$, (d) $T=0.7 \mathrm{~s}$, (e) $T=1.0 \mathrm{~s}$ and (f) $T=3.0 \mathrm{~s}$. 


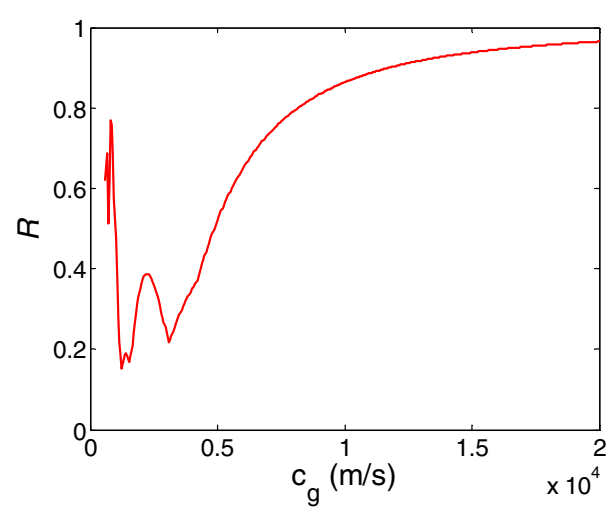

(c)

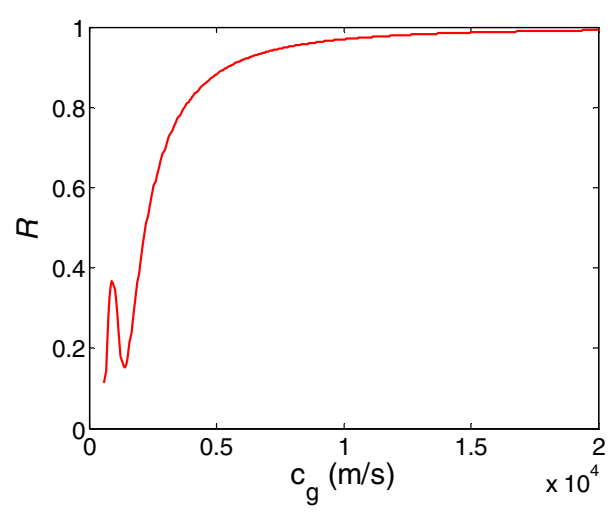

(e)

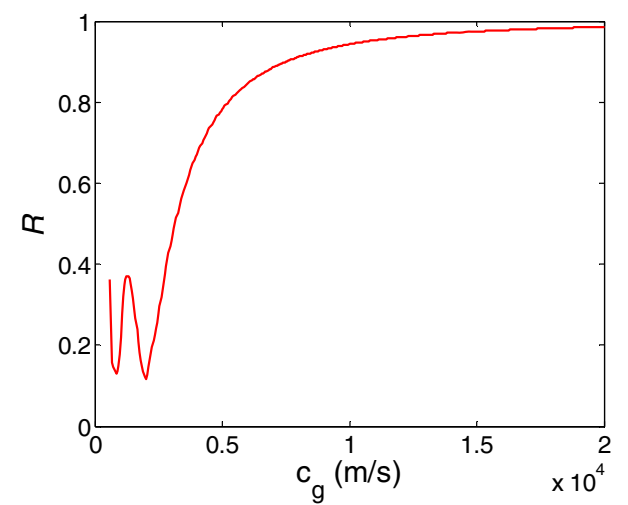

(d)

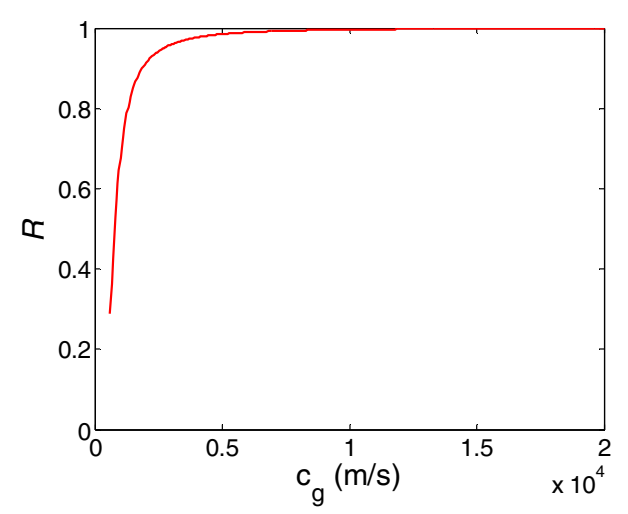

(f)

Fig. 8. (Continued)
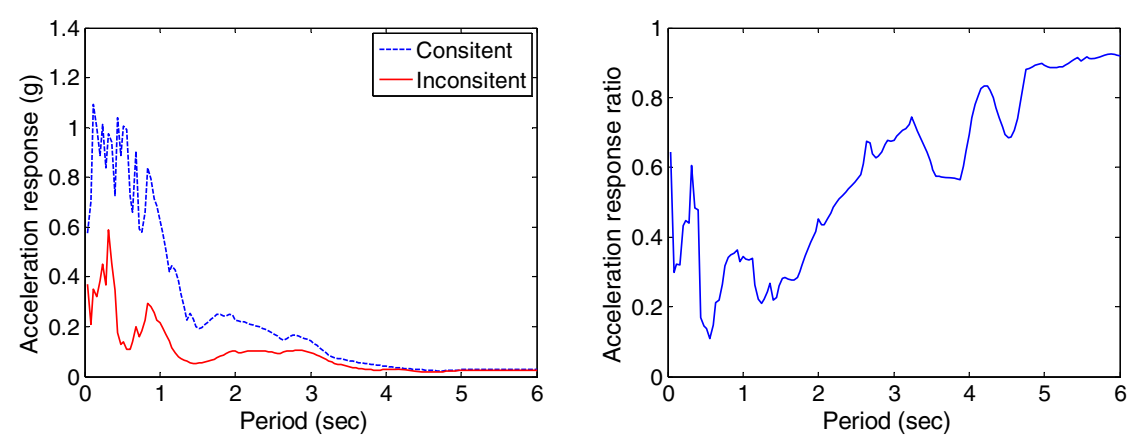

(a)

Fig. 9. Wave-passage effect on the response spectra and the response spectral ratios for different apparent velocities. (a) $c_{g}=1000 \mathrm{~m} / \mathrm{s}$, (b) $c_{g}=1500 \mathrm{~m} / \mathrm{s}$, (c) $c_{g}=2500 \mathrm{~m} / \mathrm{s}$, (d) $c_{g}=3000 \mathrm{~m} / \mathrm{s},(\mathrm{e}) c_{g}=5000 \mathrm{~m} / \mathrm{s}$, (f) $c_{g}=10000 \mathrm{~m} / \mathrm{s}$ and $(\mathrm{g}) c_{g}=20000 \mathrm{~m} / \mathrm{s}$. 
Wave-Passage Effect of Earthquake Loadings on Long Structures
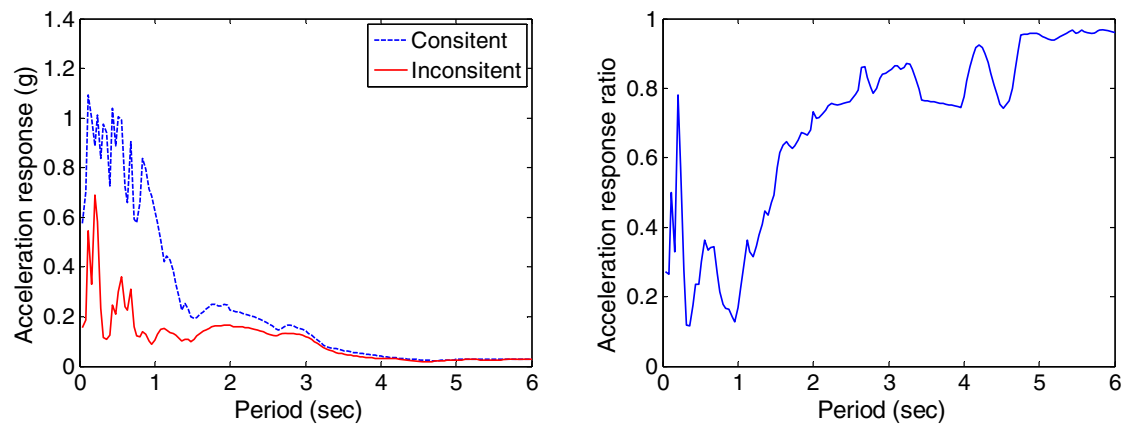

(b)
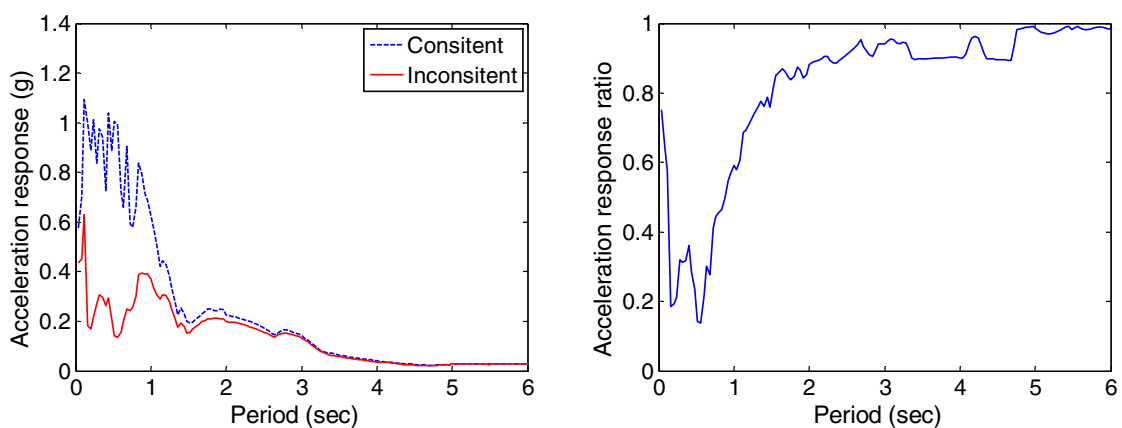

(c)
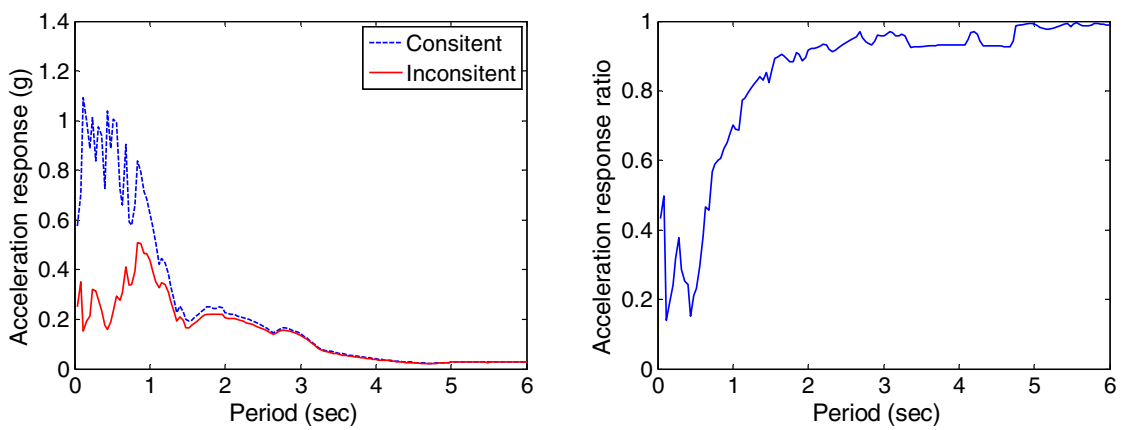

(d)

Fig. 9. (Continued) 
D. Wang et al.
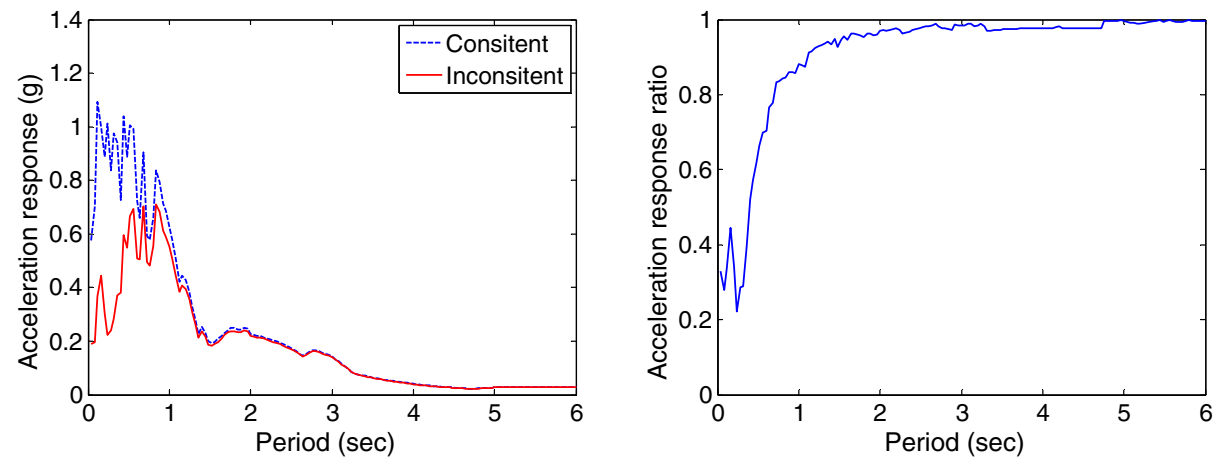

(e)
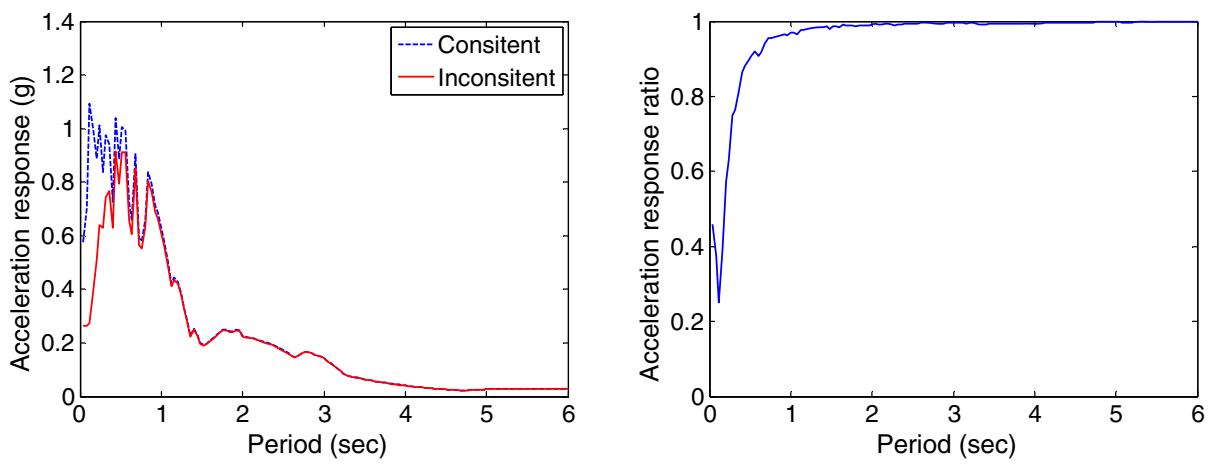

(f)
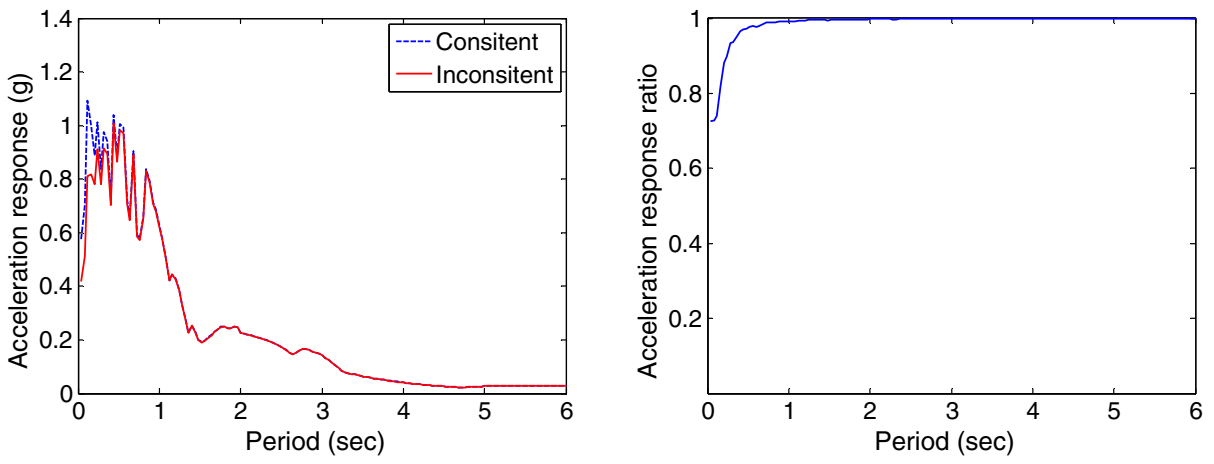

(g)

Fig. 9. (Continued) 
wave velocity range, with a mean value of about 0.5 , and gradually increases as the apparent wave velocity increases. With increasing natural period, the fluctuation range of $R$ becomes narrower and then disappears.

Figure 9 shows the wave-passage response spectra and response spectral ratios for different apparent wave velocities. Consistent with the characteristics shown in Fig. 8, the reduction of the maximum dynamic earthquake loading is remarkable and fluctuant for low apparent wave velocities and low structural natural periods, and is negligible for high apparent wave velocities or high structural natural periods.

\section{Conclusion}

The wave-passage effect on the dynamic earthquake loading on a multiply-supported SDOF system is studied. For this SDOF system, the time-domain derivation shows that the absolute acceleration response excited by the traveling seismic wave can be expressed as a series of the absolute acceleration response excited by the corresponding uniform seismic wave. The analysis of the Fourier spectrum indicates that, in the frequency domain, the wave-passage effect reduces the absolute acceleration response and the dynamic earthquake loading acting on the system.

In one numerical example, the wave-passage response spectra are computed to measure the wave-passage effect on the maximum dynamic earthquake loading for different apparent wave velocities. The results show that, consistent with the frequency-domain analysis results, the wave-passage effect reduces the maximum dynamic earthquake loading. Generally, the reduction of the maximum dynamic earthquake loading is remarkable for small structural natural periods, $T$, and gradually decreases with the increasing of $T$. The results of the response spectral computation also show that the effect of the apparent wave velocity on the dynamic earthquake loading is fluctuant and complex. The reduction of the maximum dynamic earthquake loading has a general tendency to decrease with increasing the apparent wave velocity.

An apparent limitation of this study is that the derivation is based on the SDOF system model with identical supports. For SDOF systems with nonidentical supports, for which the absolute acceleration response cannot be simply expressed as a series of the corresponding absolute acceleration response excited by the uniform seismic wave, the procedure of analysis is much more complex. It should also be noted that the wave-passage effect just causes the reduction in the absolute displacement, velocity, acceleration responses and the dynamic earthquake loading on the SDOF structure. The deformations and internal forces of structural members are not only determined by the dynamic earthquake loading, but also by the pseudostatic responses, which is caused by the deformation of the ground. Further studies are needed to find an intuitive and convenient tool to determine the comprehensive influence of the dynamic earthquake loading and the ground deformation on extended structures. 


\section{Acknowledgments}

This research was supported by National Natural Science Foundation of China (Grant Nos. 51408526 and 51308487), National Basic Research Program of China (Grant No. 2013CB834100) and Natural Science Foundation of Hebei Province (E2015203274).

\section{References}

1. J. L. Bogdanoff, J. E. Goldberg and A. J. Schiff, The effect of ground transmission time on the response of long structures, Bull. Seismol. Soc. Am. 55 (1965) 627-640.

2. A. Zerva, Spatial Variation of Seismic Ground Motions: Modeling and Engineering Applications (CRC Press: New York, 2009).

3. A. Der Kureghian and A. Neuenhofer, Response spectrum method for mulit-support seismic excitations, Earthquake Eng. Struct. Dynam. 21(8) (1992) 713-740.

4. C. H. Loh, Analysis of the spatial variation of seismic waves and ground movements from SMART-1 data, Earthquake Eng. Struct. Dynam. 13 (1985) 561-581.

5. R. S. Harichandran and E. H. Vanmarcke, Stochastic variation of earthquake ground motion in space and time, J. Eng. Mech., ASCE 112 (1986) 154-174.

6. J. E. Luco and H. L. Wong, Response of a rigid foundation to a spatially random ground motion, Earthquake Eng. Struct. Dynam. 14 (1986) 891-908.

7. H. Hao, C. S. Oliveira and J. Penzien, Multiple-station ground motion processing and simulation based on SMART-1 array data, Nuclear Eng. Design 111 (1989) 293-310.

8. C. H. Loh and S. G. Lin, Directionality and simulation in spatial variation of seismic waves, Eng. Struct. 12 (1990) 134-143.

9. C. S. Oliveira, H. Hao and J. Penzien, Ground motion modeling for Multiple-input structural analysis, Struct. Safety 10 (1991) 79-93.

10. N. A. Abrahamson, Spatial variation of multiple support inputs, in Proc. 1st U. S. Seminar on Seismic Evaluation and Retrofit of Steel Bridges, A Caltrans and University of California at Berkeley Seminar, San Francisco, CA (1993).

11. A. Bayraktar, A. A. Dumanoglu and Y. Calayir, Asynchronous dynamic analysis of dam reservoir foundation systems by the Langrangian approach, Comput. Struct. 58 (1996) 925-935.

12. A. Bayraktar and A. A. Dumanoglu, The effect of the asynchronous ground motion on hydrodynamic pressures, Comput. Struct. 68 (1998) 271-282.

13. G. Monti, C. Nuti and P. E. Pinto, Nonlinear response of bridges under multi-support excitation, J. Struct. Eng., ASCE 122 (1996) 1147-1159.

14. Z. Zembaty and S. Krenk, Spatial seismic excitations and response spectra, J. Eng. Mech., ASCE 119 (1993) 2449-2459.

15. M. I. Todorovska and M. D. Trifunac, A note on the propagation of earthquake waves in building with soft first floor, J. Eng. Mech., ASCE 116 (1990) 892-900.

16. R. S. Jalali, M. D. Trifunac, G. G. Amiri and M. Zahedi, Wave-passage effects on strength-reduction factors for design of structures near earthquake faults, Soil Dynam. Earthquake Eng. 27 (2007) 703-711.

17. R. S. Jalali and M. D. Trifunac, A note on strength-reduction factors for design of structures near earthquake faults, Soil Dynam. Earthquake Eng. 28 (2008) 212-222.

18. M. J. O'Rourke, M. C. Bloom and R. Dobry, Apparent propagation velocity of body waves, Earthquake Eng. Struct. Dynam. 10 (1982) 283-294. 
19. H. Boissières and E. H. Vanmarcke, Estimation of lags for a seismograph array: Wave propagation and composite correlation, Soil Dynam. Earthquake Eng. 14 (1995) 5-22.

20. S. Quayyum, I. M. Nazmul and M. Iasmin and K. M. Amanat, Effects of randomly distributed infill on columns of reinforced concrete frames with soft ground storey, Int. J. Struct. Stab. Dynam. 10(3) (2011) 555-569.

21. H. Degee, B. Rossi and D. Jehin, Geometrically nonlinear analysis of steel storage racks submitted to earthquake loading, Int. J. Struct. Stab. Dynam. 11(5) (2011) 949-967. 